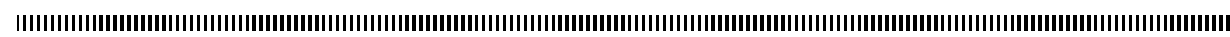

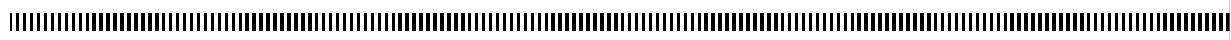

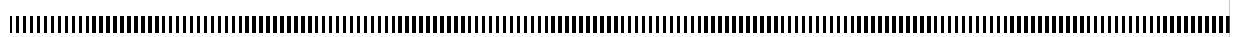

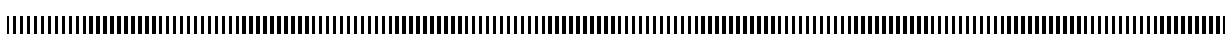

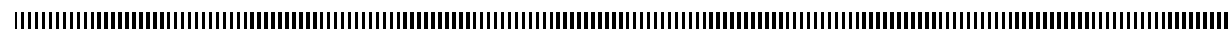

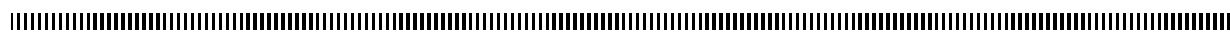
|IIIIIIIIIIIIIIIIIIIIIIIIIIIIIIIIIIIIIIIIIIIIIIIIIIIIIIIIIIIIIIIIIIIIIIIIIIIIIIIIIIIIIIIIIIIIIIIIIIIIIIIIIIIIIIIIIIIIIIIIIIIIIIIIIIIIIIIIIIIIIIIIIIIIIIIIIIIIIIIIIIIIIIIIIIIIIIIIIIIIIIIIIIIII

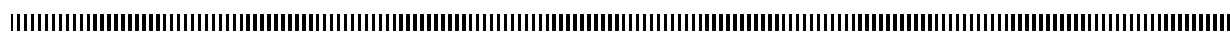

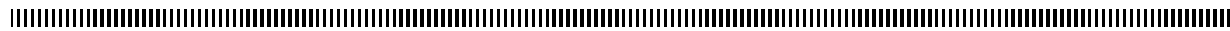

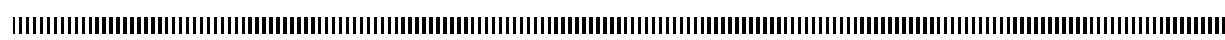

\title{
Vers une métrique de description objective d'une sensation subjective
}

\author{
Sonia Ouni ${ }^{1,2}$, Ezzeddine Zagrouba ${ }^{1}$, Majed Chambah ${ }^{2}$, Michel Herbin ${ }^{2}$ \\ ${ }^{1}$ Equipe de recherche SIVREC, Institut Supérieur Informatique de Tunis \\ Abou Raihane Bayrouni 2080, Ariana, TUNISIA \\ ${ }^{2}$ CReSTIC, Université de Reims Champagne Ardenne \\ Rue des crayères BP 103551687 REIMS Cedex 2 \\ sonia.ouni@etudiant.univ-reims.fr / ezzeddine.zagrouba@fsm.rnu.tn \\ majed.chambah@univ-reims.fr/michel.herbin@univ-reims.fr \\ [|+|
}

RÉSUMÉ. L'étude du système visuel Humain (SVH) est primordiale pour quantifier la qualité d'une image. Plusieurs travaux ont proposé des métriques de différences de couleurs qui effectuent des différences locales pixel par pixel. Par contre, le système visuel humain est plutôt sensible à une qualité globale. Dans cet article, nous proposons une nouvelle métrique objective de différence couleur basée sur les caractéristiques du système visuel humain. La métrique proposé est appelée Delta $E$ Globale notée : $\widetilde{E}_{G}$. Quelques expérimentations et évaluations montrent la performance de cette métrique et sa corrélation avec la vision du SVH.

ABSTRACT. In the last decade, several image and video quality metrics have been proposed, which incorporate perceptual quality measures by considering the HVS characteristics. All this metrics do the differences pixel to pixel in image. Therefore a local fidelity of the colour is defined. However, the human visual system is rather sensitive to a global quality. In this paper, we propose a new objective full reference quality metric for colour images called Global Delta $E$ and noted: $\Delta \widetilde{E}_{G}$. This metric is based on human visual system properties in order to obtain the best correspondence with judgments. Some experimentations and assessments prove the performance of our metrics and that interrelationship with the SVH.

MOTS-CLÉS : système visuel Humain, différence couleur, qualité, Delta E globale.

KEYWORDS: Human Visual System, colour difference, Quality, Global Delta E.

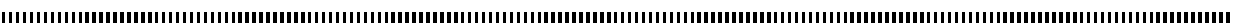

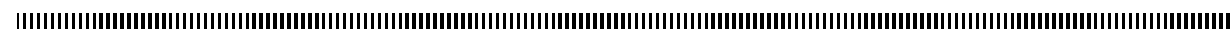

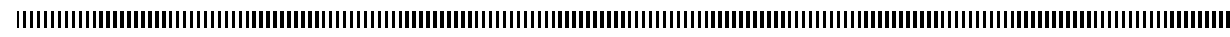

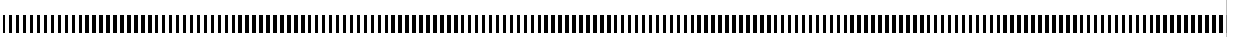

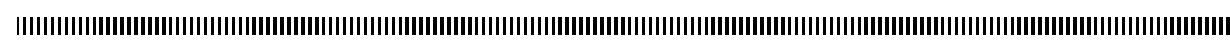

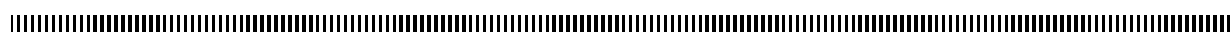

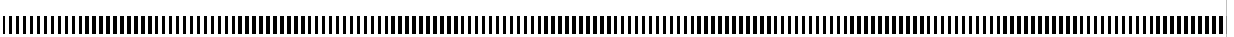

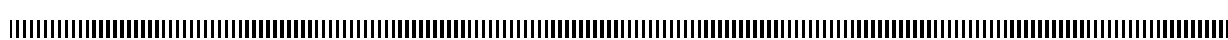

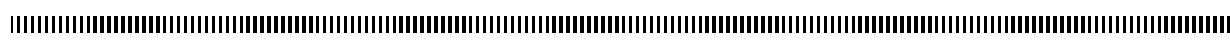

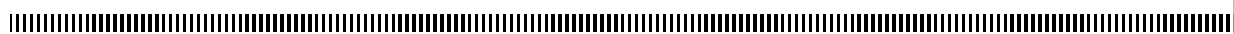

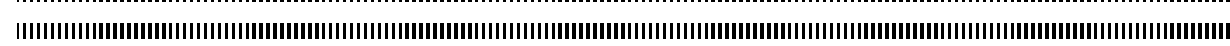




\section{Introduction}

La télévision, internet, le DVD mais aussi les services téléphonie mobile ou les systèmes multimédias sont autant d'exemples d'applications pour lesquelles des techniques de traitement d'images sont développées. D'où l'intérêt d'évaluer la qualité des images numériques afin d'être en mesure d'estimer l'efficacité des méthodes développées.

La mesure de la qualité visuelle des images ou de la vidéo s'avère très utile dans plusieurs applications en imagerie : codage [1][12][19], restauration[8], imagerie médicale [11]. Les méthodes développées dans ces domaines peuvent être classées en deux grandes approches. La première, appelée classiquement évaluation subjective, est basée sur l'utilisation d'un groupe d'observateurs humains qui doivent noter la qualité des images dans les conditions d'observation appropriées [16]. Cette approche reste la plus utilisée et la plus efficace dans la plupart des domaines, comme la qualité d'impression, la compression et la restauration des films cinématographiques, mais dernière est complexe et chronophage.

La deuxième approche est l'évaluation objective qui utilise des mesures pour quantifier la qualité des images. Puisqu'elle est automatisée, l'évaluation objective est moins coûteuse que l'évaluation subjective. De plus, elle peut être effectuée en temps réel puisqu'elle ne nécessite pas d'interaction avec l'utilisateur. L'évaluation objective de la qualité peut se décliner en trois catégories, celles qui sont à référence totale (utilisation complète de l'image originale), appelées aussi « Full Reference », celles qui utilisent quelques paramètres de la référence, appelées aussi «Reduced Reference » ou méthodes à référence réduite [17] et celles qui n'utilisent aucune référence [18]. Les méthodes «Reduced Reference » sont souvent utilisées dans le cadre de la transmission des images. Les méthodes «Sans Référence » se basent sur la connaissance a priori de la nature des dégradations ou/et des images. Les méthodes sans référence et avec référence réduite ne feront pas l'objet de cet article.

Dans l'approche avec référence complète, plusieurs études ont donc tenté de proposer une méthode de calcul de différences de couleurs qui prennent en compte la perception des couleurs. Le problème est que ces mesures effectuent des différences pixel à pixel pour définir une fidélité locale de la couleur. Or, le système visuel humain est plutôt sensible à une qualité globale.

Dans ce papier, nous proposons une nouvelle métrique qui prend en considération les caractéristiques du système visuel humain. La mesure proposée se place dans l'hypothèse des méthodes avec référence complète : nous nous plaçons dans le cadre de l'usage des images numériques codées et restaurées. Notre préoccupation est d'être le plus indépendant possible de la méthode du traitement utilisée, de surmonter le problème de la mesure des dégradations localisées et concentrées, d'être indépendant du type d'image et de se rapprocher de la perception du système visuel humain.

\section{A R I M A}


L'originalité de cette métrique objective est la prise en compte de la vision globale de l'image.

La suite de ce papier est organisée comme suit : la section 2 est dédiée aux métriques de différence de couleur. Les détails de la métrique proposée sont donnés dans la section 3. Dans la section 4, nous validons notre approche par quelques expérimentations et évaluations. Enfin, nous terminerons par une conclusion et des perspectives.

\section{Métriques de différence de couleur}

Dans la littérature, de nombreux travaux ont tenté de proposer une méthode de calcul de différences de couleurs qui prennent en compte la perception des couleurs [2].

Le modèle de couleur CIE $\mathrm{L}^{*} \mathrm{a} * \mathrm{~b} *$ étant proche de notre perception visuelle, la mesure des différences chromatiques visuelles revient à mesurer des distances euclidiennes entre couleurs dans ce modèle. Le Delta E [6] est la formule de mesure des distances «perceptuelles» entre deux couleurs.

$$
\Delta E^{*}=\sqrt{\Delta L^{* 2}+\Delta a^{* 2}+\Delta b^{* 2}}
$$

En 1976, McLaren [7] introduit des facteurs de pondération pour chaque composante dans sa formule de différence de couleurs. McLaren combine l'équation proposée par Mc-Donald [14] qui simplifie l'équation complexe de différence de couleurs en donnant deux fois plus de poids à l'information de teinte par rapport aux informations de luminance et de chromaticité. L'équation obtenue pour la différence de couleurs est la suivante :

$$
\Delta E_{(M C)^{2}}=\sqrt{\frac{(\Delta L)^{2}+(\Delta C)^{2}+(2 \Delta H)^{2}}{1+0.02 C}}
$$

En 1979, McDonald propose d'utiliser des facteurs non linéaires dans les équations de différences pour donner la formule $\Delta$ EJPC79. Des facteurs de pondération sont appliqués sur la différence de luminance (SL), de chromaticité (SC) et de teinte (SH). La métrique résultante est donnée par l'équation (3), oừ $\mathrm{L}, \mathrm{C}$ et $\mathrm{h}$ sont calcules a partir des formules de Adams- Nickerson [3] :

$$
\Delta E_{J P C 97}=\sqrt{\left(\frac{\Delta L}{S_{L}}\right)^{2}+\left(\frac{\Delta C_{a b}}{S_{C}}\right)^{2}+\left(\frac{\Delta H_{a b}}{S_{H}}\right)^{2}}
$$

Dans cette équation $\mathrm{S}_{\mathrm{L}}$ dépend de $\mathrm{L}, \mathrm{S}_{\mathrm{C}}$ dépend de $\mathrm{C}$ et $\mathrm{S}_{\mathrm{H}}$ dépend de $\mathrm{h}$.

En 1984, Clarke et al. [5][13] proposent l'équation de différence de couleur CMC(1 :c) basée sur le JPC79. Deux facteurs de pondération supplémentaires (1 et c) sont choisis en fonction de la matière réfléchissant la lumière. L'équation de différence s'écrit alors : 


$$
\Delta E_{C M C(l: c)}=\sqrt{\left(\frac{\Delta L}{l S_{L}}\right)^{2}+\left(\frac{\Delta C_{a b}}{c S_{C}}\right)^{2}+\left(\frac{\Delta H_{a b}}{S_{H}}\right)^{2}}
$$

En 1987, Luo et Rigg [9] ont défini l'équation de différence de couleur $\Delta \mathrm{E}_{\mathrm{BFD}(1: c)}$ pour corriger les défauts de l'équation CMC(1:c) dans le bleu. En 1994, la Comité Internationale d'Eclairage (CIE) préconise l'utilisation d'une nouvelle équation pour les différences de couleurs basée sur les travaux de Berns et al. [15]. La formulation de cette équation est similaire à la structure $\mathrm{CMC}(1$ :c) avec des facteurs de pondération simplifiés :

$$
\Delta E_{94}=\sqrt{\left(\frac{\Delta L_{a b}^{*}}{K_{L} S_{L}}\right)^{2}+\left(\frac{\Delta C_{a b}^{*}}{K_{C} S_{C}}\right)^{2}+\left(\frac{\Delta H_{a b}^{*}}{K_{H} S_{H}}\right)^{2}}
$$

où̀ $\mathrm{K}_{\mathrm{L}}, \mathrm{K}_{\mathrm{C}}$ et $\mathrm{K}_{\mathrm{H}}$ sont des facteurs de pondération dépendant des conditions expérimentales. La CIE recommande de fixer tous les facteurs de pondération à 1, sauf pour les applications de l'industrie textile.

En 1999, Kim et Nobbs [4] proposent une étude sur la luminance, la chromaticité et la teinte basée sur l'équation $\Delta \mathrm{E}_{94}$. Ils ont mis en évidence que la tolérance de cette formule pour des luminosités faibles ou importantes est plus grande que pour des luminosités moyennes. Le facteur de pondération $\mathrm{S}_{\mathrm{L}}$ dans la formule $\Delta \mathrm{E}_{94}$ se révèle donc insuffisant pour décrire ce phénomène. De plus, ils mettent en évidence l'insuffisance des critères de pondération $S_{C}$ et $S_{H}$ pour décrire les phénomènes sur la chromaticité.

En 2000, la CIE propose une dernière équation pour le calcul de différence de couleurs basée sur les travaux de Luo et al. [10]. Comparée à l'équation $\Delta \mathrm{E}_{94}$, la formulation $\Delta \mathrm{E}_{2000}$ est plus complexe, mais elle donne de meilleurs résultats. La méthode se décompose en quatre étapes à partir des valeurs CIE L ${ }^{*}{ }^{*} b^{*}$ :

\section{- Etape 1 : Calcul de C}

La première étape correspond au calcul de la chromacité

$$
C=\sqrt{a^{2}+b^{2}}
$$

\section{- Etape 2 : Calcul de $a^{\prime}, C^{\prime}$ et $h^{\prime}$}

La deuxième étape a pour objectif de rajuster les échelons $a^{*}$ pour les teintes neutres et ainsi corriger les définitions de la chromacité $C$ et de la teinte $h$ :

$$
\begin{gathered}
a^{\prime}=(1+G) a \\
C^{\prime}=\sqrt{a^{\prime 2}+b^{2}} \\
h^{\prime}=\arctan \left(\frac{b}{a^{\prime}}\right)
\end{gathered}
$$

\section{A R I M A}


avec :

$$
G=0.5\left(1-\sqrt{\frac{\bar{C}^{7}}{\bar{C}^{7}+25^{7}}}\right)
$$

$\bar{C}$ correspond à la moyenne arithmétique des valeurs des $\mathrm{C}$ des deux couleurs.

\section{- Etape 3 : Calcul de $\Delta L^{\prime}, \Delta C^{\prime}$ et $\Delta H^{\prime}$}

L'étape 3 définit des écarts de luminance, de teinte et de chromacité :

$$
\begin{gathered}
\Delta L=L_{B}-L_{S} \\
\Delta C^{\prime}=C_{B}^{\prime}-C_{S}^{\prime} \\
\Delta h^{\prime}=h_{B}^{\prime}-h_{S}^{\prime} \\
\Delta H^{\prime}=2 \sin \frac{\Delta \mathrm{h}^{\prime}}{2} \times \sqrt{C_{S}^{\prime} C_{B}^{\prime}}
\end{gathered}
$$

- Etape 4 : Calcul de l'écart colorimétrique

$$
\Delta E_{2000}=\sqrt{\left(\frac{\Delta L}{S_{L}}\right)^{2}+\left(\frac{\Delta C}{S_{C}}\right)^{2}+R_{t}\left(\frac{\Delta C^{\prime}}{S_{C}} \times \frac{\Delta H^{\prime}}{S_{H}}\right)^{2}}
$$

A l'heure actuelle, un groupe de travail de la CIE cherche encore à améliorer cette formule pour une meilleure prise en compte des différents phénomènes de l'apparence couleur. Les relations de distances proposées effectuent des calculs locaux dans une image. Il est donc intéressant d'introduire, en amont dans les calculs de différences, les caractéristiques du système visuel humain, pour aboutir à une métrique plus proche de la réalité visuelle.

\section{Vers une description objective d'une sensation subjective}

Les performances d'un critère objectif de qualité d'image s'estiment par sa capacité à produire une mesure de qualité aussi proche que possible de celle du jugement humain. Une des caractéristiques importantes du SVH est la vision globale de l'image. Dans cette métrique, nous allons modéliser cette sensation subjective par une description objective.

Le modèle utilisé dans cette métrique de qualité est décrit dans la Figure 1. Il repose tout d'abord sur une transformation du triplet $(\mathrm{R}, \mathrm{V}, \mathrm{B})$ dans l'espace uniforme XYZ, ensuite dans l'espace $\mathrm{L}^{*} \mathrm{a}^{*} \mathrm{~b}^{*}$ et ceci pour calculer la différence entre un pixel de l'image originale et un pixel de l'image dégradée. 


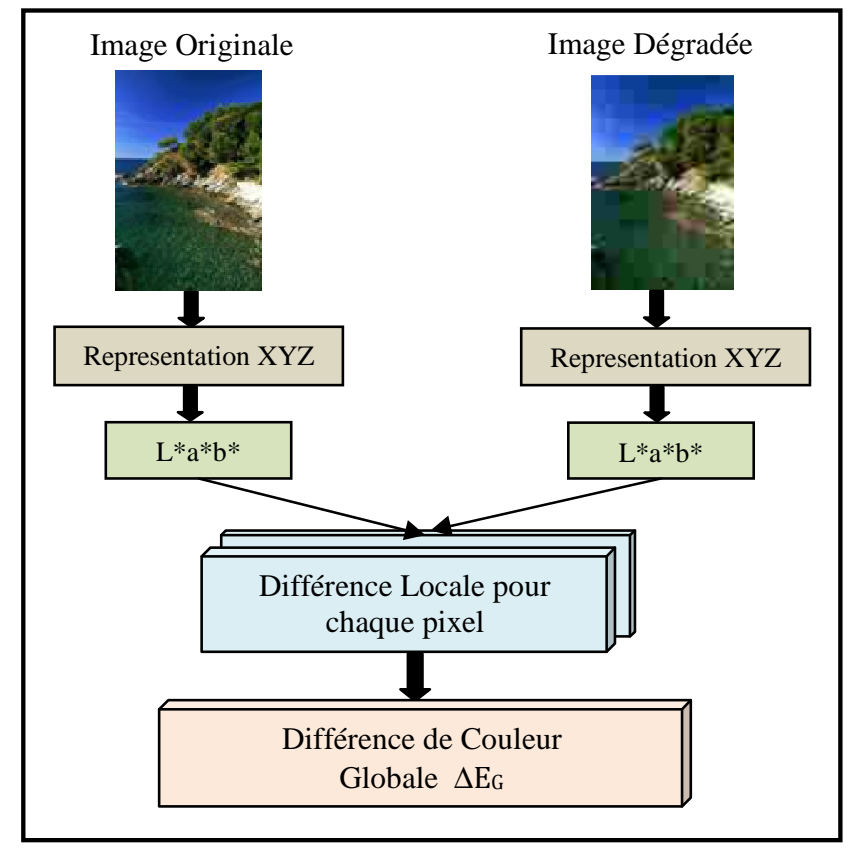

Figure 1. Modèle proposé.

Soit $\mathrm{X}=\left\{\mathrm{x}_{\mathrm{i}, \mathrm{j}} \mid \mathrm{i}=1, \ldots, \mathrm{M} ; \mathrm{j}=1, \ldots, \mathrm{N}\right\}$ et $\mathrm{Y}=\left\{\mathrm{y}_{\mathrm{i}, \mathrm{j}} \mid \mathrm{i}=1, \ldots, \mathrm{M} ; \mathrm{j}=1, \ldots, \mathrm{N}\right\}$ respectivement l'ensemble des pixels de l'image originale et ceux de l'image dégradée. Le calcul de la distance est réalisé par notre nouvelle métrique (16) basée essentiellement sur une comparaison locale au niveau pixel (figure 4).

$$
\Delta \tilde{E}(i, j)=\frac{\Delta E(i, j)+\sum_{k=1}^{N} \sum_{l=1}^{M} P(k, l) \Delta E(k, l)}{1+\sum_{k=1}^{N} \sum_{l=1}^{M} P(k, l)} \quad \begin{array}{ll}
\text { avec } \mathrm{k} \in[1 . . \mathrm{N}], l \in[1 . . \mathrm{M}] \\
\text { et } \mathrm{k} \neq \mathrm{i} \text { et } \mathrm{l} \neq \mathrm{j}
\end{array}
$$

Dans cette distance on introduit la notion de voisinage. L'influence des pixels voisins dans le calcul de $\Delta \widetilde{E}(i, j)$ s'attenue en fonction de leurs éloignements. Cela est réalisé avec la pondération $P$.

$$
P(k, l)=\frac{1}{d^{2}((k, l),(i, j))} \text { avec } \mathrm{l} \neq \mathrm{i} \text { et } \mathrm{k} \neq \mathrm{j}
$$

avec $d$ est la distance entre les pixels.

\section{A R I M A}




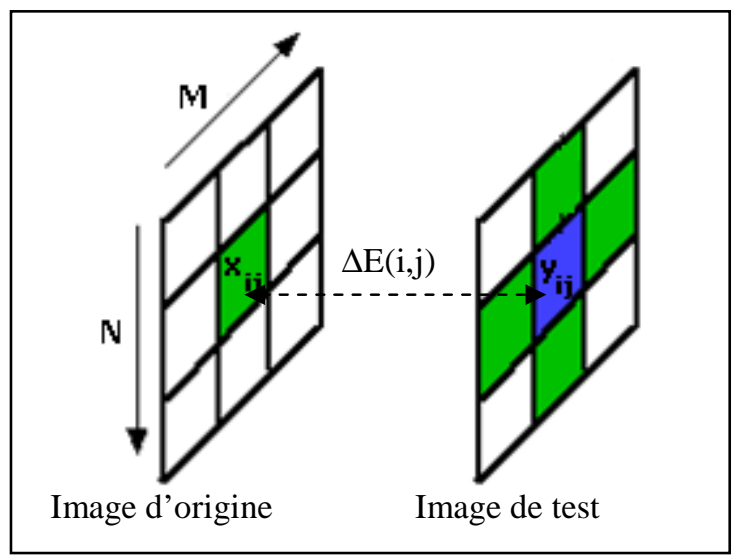

Figure 2. Différence Locale pour chaque pixel.

Enfin la distance globale entre les deux images est définie comme suit :

$$
\Delta \tilde{E}_{G}=\frac{1}{\mathrm{M} \times \mathrm{N}} \sum_{\mathrm{i}=1}^{\mathrm{N}} \sum_{\mathrm{j}=1}^{\mathrm{M}} \Delta \tilde{\mathrm{E}}(\mathrm{i}, \mathrm{j})
$$

Cette métrique fait intervenir la notion d'adjacence dans le calcul de différence de couleur ceci permet de traduire une sensation subjective du SVH qui est la vision globale de l'image.

\section{Expérimentations}

Dans cette section, nous représentant nos expérimentations afin de mettre en œuvre l'efficacité de la métrique proposée. Pour ce faire, nous avons utilisé un ensemble d'images représentons des thèmes variés (figure 3 ).

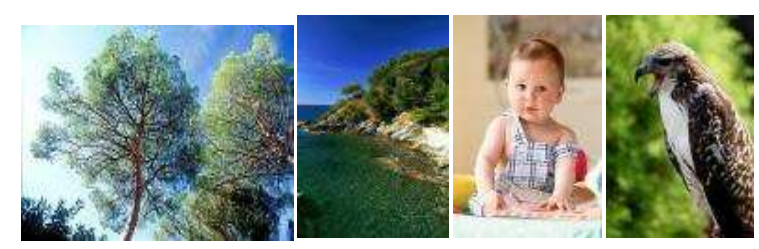

Figure 3. Exemple d'images de la base. 
Deux scénarios d'expériences sont réalisés. Le premier scénario est basé sur un ensemble d'images compressées avec $\mathrm{Jpeg}_{2000}$ avec plusieurs types de dégradation. La qualité de ces images a été évaluée avec $\Delta \mathrm{E}_{2000}$ et la métrique $\Delta \mathrm{E}_{\mathrm{G}}$. Le deuxième scénario consiste à évaluer des images restaurées et étudier la corrélation entre le MOS et les métriques objectives.

Dans le domaine de la compression nous avons établit les expérimentations suivantes. Comme le montre la figure 4 , l'écart $\Delta \mathrm{E}_{2000}$ moyen entre l'image originale et sa version compressée (figure 4-b) est plus important que l'écart entre cette même image et une version ou un élément du contenu a subi une modification de teinte significative (figure 4-c). Or, le système visuel humain est plus sensible à la modification de la couleur d'un élément de l'image. Cependant avec la métrique proposée $\Delta \mathrm{E}_{\mathrm{G}}$ nous obtenons un écart plus important avec l'image modifiée plus que l'image compressée. Ceci montre que notre métrique corrèle bien avec la vision de l'être humain.

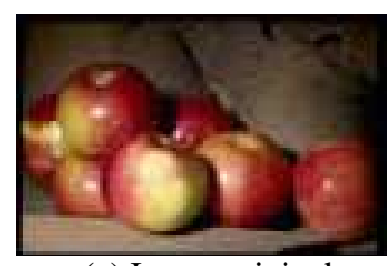

(a) Image originale

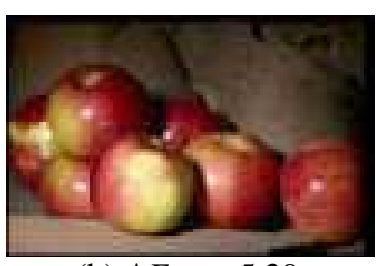

(b) $\Delta E_{2000}=5.29$ $\Delta E_{\mathrm{G}}=6.42$

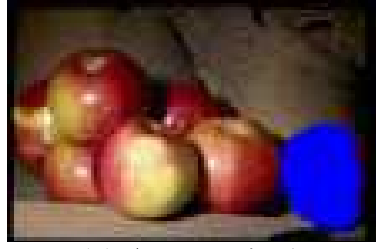

(c) $\Delta E_{2000}=4.61$ $\Delta E_{\mathrm{G}}=16.33$

Figure 4. Exemple de mesures de différence en utilisant la métrique $\Delta E_{2000}$ et $\Delta E_{\mathrm{G}}$ entre une image originale et deux versions modifiées : une compression JPEG(b) et une modification de couleur sur une pomme de l'image(c).

La figure 5, confirme l'efficacité de notre métrique qui permet d'obtenir des résultats meilleurs que le $\Delta \mathrm{E}_{2000}$. Visuellement on remarque que l'écart entre l'image originale et les images modifiées (c) et (d) est plus élève que cette même image et sa version compressée ce qui est le cas pour notre métrique $\Delta \mathrm{E}_{\mathrm{G}}$ contrairement à $\Delta \mathrm{E}_{2000}$.

\section{A R I M A}




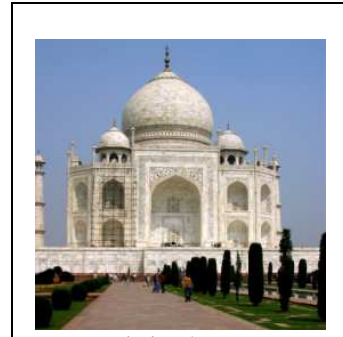

(a) original Image

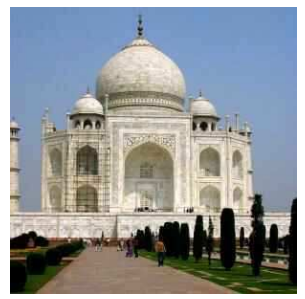

(b) $\Delta E_{2000}=3.23$ $\Delta E_{\mathrm{G}}=3.80$

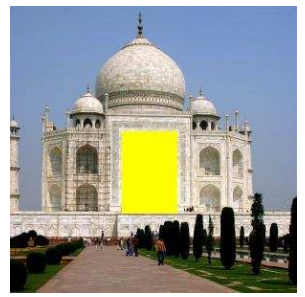

(c) $\Delta E_{2000}=2.20$ $\Delta E_{\mathrm{G}}=5.53$

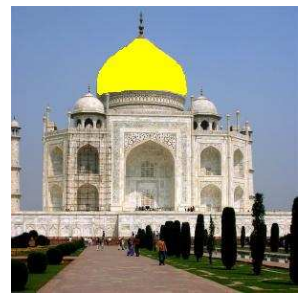

(d) $\Delta E_{2000}=1.60$ $\Delta E_{\mathrm{G}}=4.26$

Figure 5. Exemple de mesure de différence en utilisant la métrique $\Delta E_{2000}$ et $\Delta E_{\mathrm{G}}$ entre une image originale et deux versions modifiées : une compression JPEG(b) et une modification de couleur sur une partie de l'image(c) et (d).

La figure 6, illustre une comparaison de l'erreur mesurée due à la compression. Cette figure permet de comparer notre approche $\Delta \mathrm{E}_{\mathrm{G}}$ avec le $\Delta \mathrm{E}_{2000}$.

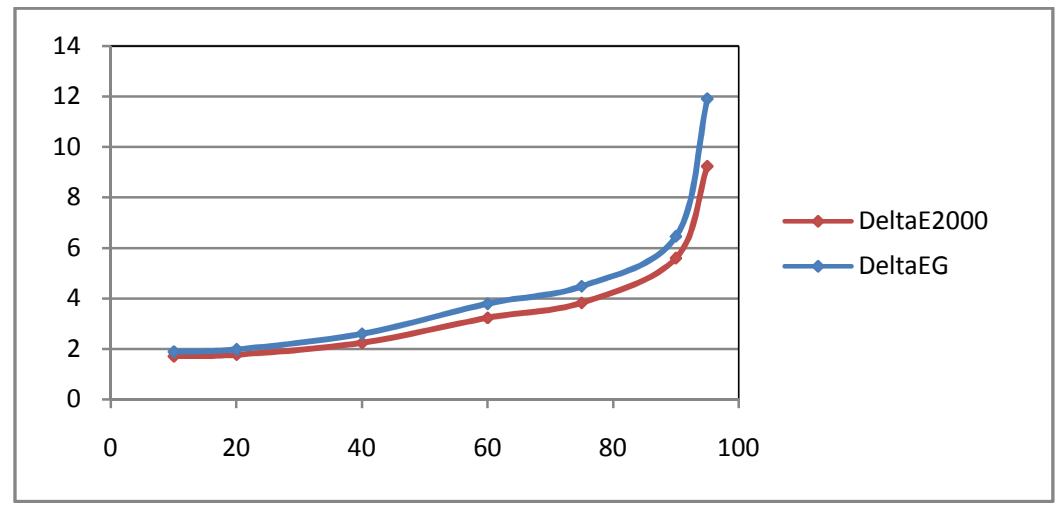

Figure 6. Mesures de fidélité pour différents taux de compression.

Nous remarquons que pour différents taux de dégradation de compression notre métrique $\Delta \mathrm{E}_{\mathrm{G}}$ produit des résultats meilleurs que le $\Delta \mathrm{E}_{2000}$.

Dans le deuxième scénario, nous appliquons notre métrique dans un contexte de restauration d'images et nous étudions la corrélation entre MOS et les métriques objective $\Delta \mathrm{E}_{2000}$ et $\Delta \mathrm{E}_{\mathrm{G}}$. Nous avons demandé à vingt observateurs d'évaluer la qualité perçue d'un ensemble d'images. La restauration d'images remédie aux problèmes d'affadissement des couleurs. Ceci est réalisé avec la méthode Automatique d'Égalisation de la Couleur (ACE) [8]. La figure suivante présente un exemple de restauration d'image. 


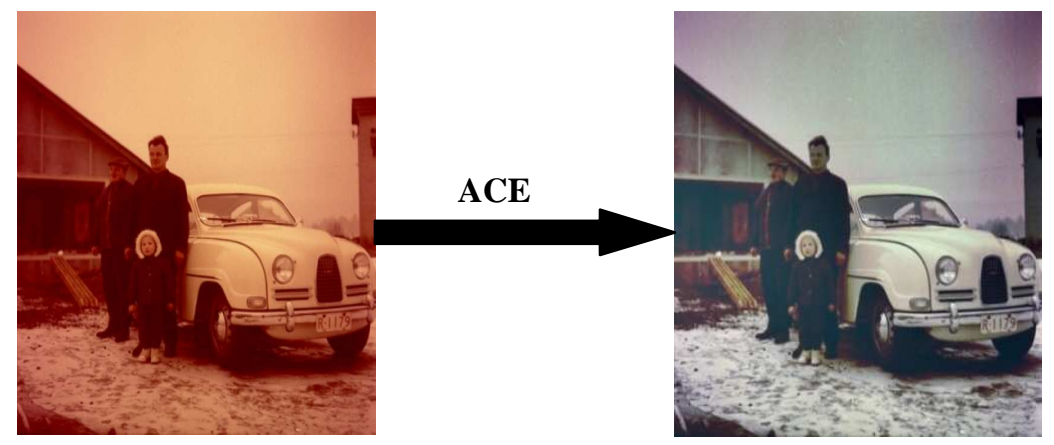

Figure 7. Exemple de restauration d'image.

Dans notre cas nous allons adopter durant les tests subjectifs la méthodologie des méthodes comparatives. Celles ci présentent l'image originale et l'image à juger tout en utilisant soit une échelle continue, soit une échelle par catégories. L'originalité de ces méthodes provient de l'échelle utilisée qui implique la présence, et parfois l'intensité, de différences perceptibles, comme indiqué sur la figure 8 qui représente une échelle par catégorie (de "beaucoup mieux" à "beaucoup moins bon").

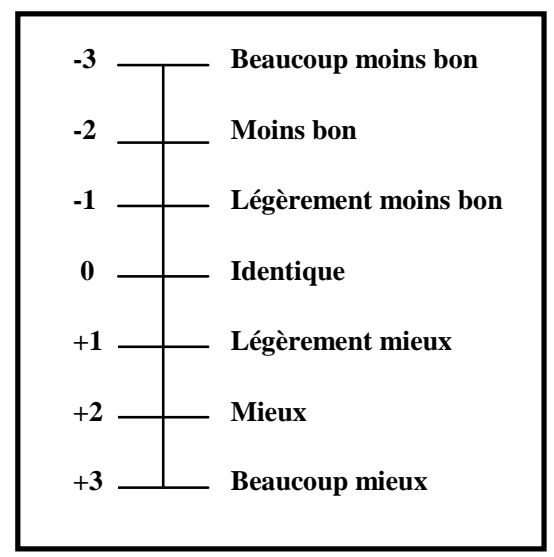

Figure 8. Echelle comparative de I'I.T.U.

Les méthodes comparatives qui utilisent une échelle continue sont basées sur le même principe qu'une échelle par catégories : la note médiane correspond à une qualité équivalente entre les deux images présentées et chaque coté de cette médiane

\section{A R I M A}


correspond à la préférence de l'une des deux images. Les notes sur l'échelle continue appartiennent à un intervalle $([-3,+3]$ par exemple) plutôt que d'être limitées à quelques catégories.

L'image originale et l'image restaurée sont présentées dans un ordre aléatoire. Les observateurs ne sont pas informés laquelle est l'originale ou la filtrée. En effet, chaque observateur est demandé de répondre à chacune des questions et il doit choisir la meilleure image parmi la paire d'images présente actuellement. Il doit noter cette paire selon l'échelle qui débute de "beaucoup mieux" jusqu'au " beaucoup moins bon ". Les questions proposées aux observateurs consistent à évaluer les points suivants :

1) le contraste de la première image par rapport à la seconde

2) la naturalité de la première image par rapport à la seconde

3) la qualité globale de la première image par rapport à la seconde (contraste, couleur)

4) la diversité chromatique de la première image par rapport à la seconde (diversité chromatique)

5) la vivacité des couleurs de la première image par rapport à la seconde (degré de coloration)

6) le niveau de préférence personnelle, ce qui revient à répondre à la question quelle image est plus proche à votre préférence personnelle?

Pour chaque paire d'images et pour chaque observateur, nous calculons les Mean Opinion Scores (MOS) relatives aux caractéristiques de contraste, de naturalité, de qualité, de diversité chromatique, de vivacité et de préférence.

$$
\operatorname{MOS}_{j k}=\frac{1}{N_{o b s}} \sum_{i=1}^{N o b j} N s u b j_{j k}(i)
$$

avec :

$\mathrm{N}_{\text {obj }}$ : nombre d'observateurs ayant noté l'image originale $\mathrm{j}$ subissant la $\mathrm{k}^{\text {ieme }}$ dégradation,

$\mathrm{N}_{\text {subjjk }}(\mathrm{i})$ : note fournie par l'observateur i ayant noté l'image originale $\mathrm{j}$ subissant la $\mathrm{k}^{\text {ieme }}$ dégradation.

$\mathrm{Si}$ un observateur fournit plusieurs notes pour l'image originale $\mathrm{j}$ subissant la $\mathrm{k}^{\text {ieme }}$ dégradation, $\mathrm{N}_{\text {subjjk }}(\mathrm{i})$ désigne la valeur moyenne de ces notes. 


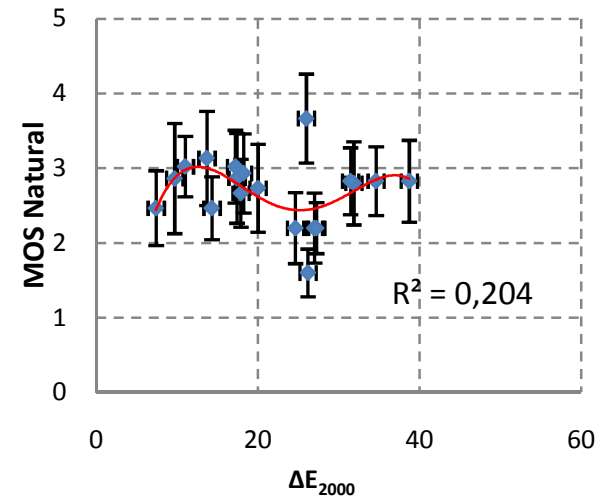

a. Corrélation entre $\Delta E_{2000}$ et MOS Natural.

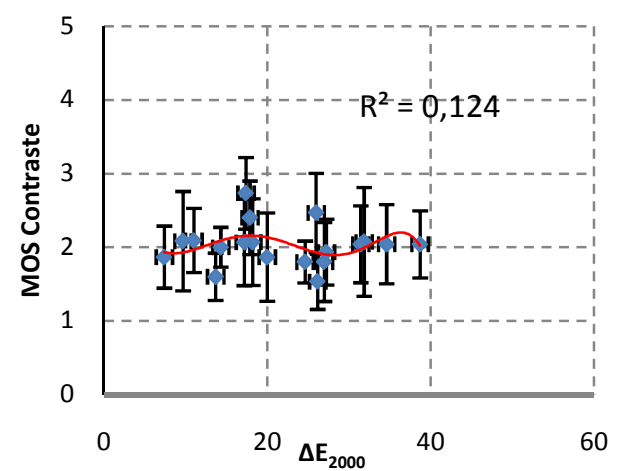

c. Corrélation entre $\Delta E_{2000}$ et MOS Contraste.

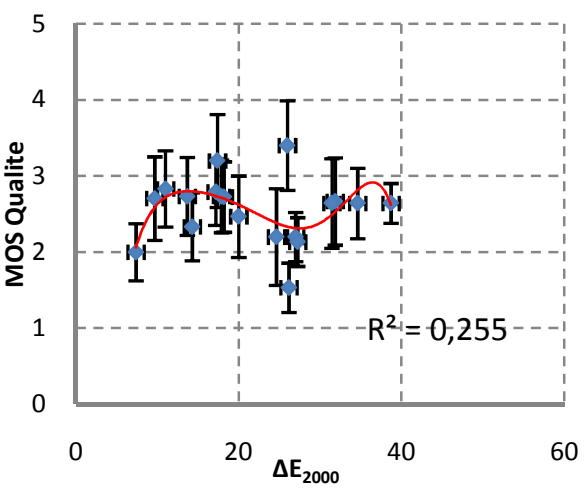

e. Corrélation entre $\Delta E_{2000}$ et MOS Qualité.

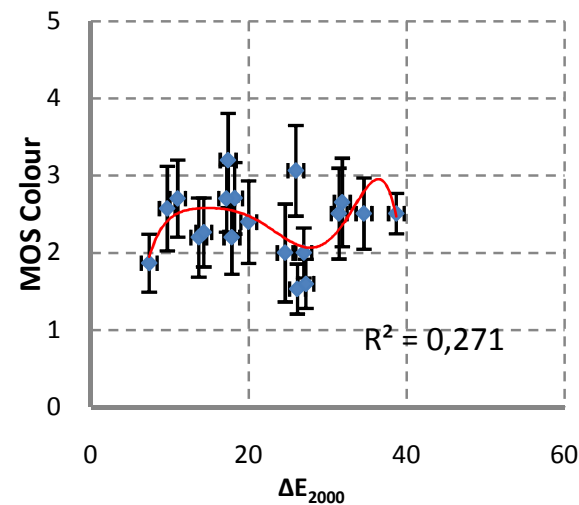

b. Corrélation entre $\Delta E_{2000}$ et MOS Couleur.

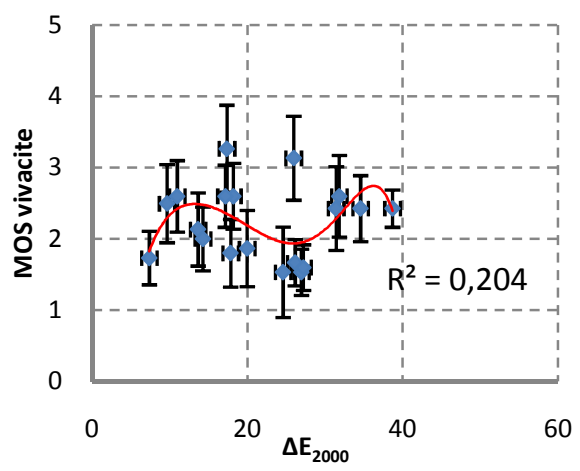

d. Corrélation entre $\triangle E 2000$ et MOS Vivacité.

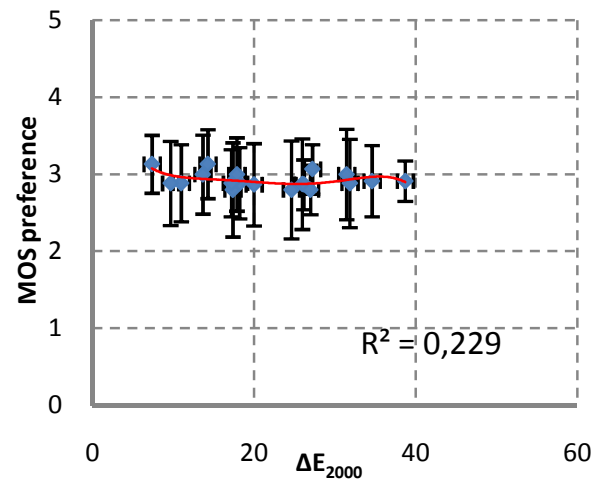

f. Corrélation entre $\Delta E_{2000}$ et MOS préférence.

Figure 9. Corrélation entre le Mean Opinion Scores (MOS) et le $\Delta E_{2000}$. 


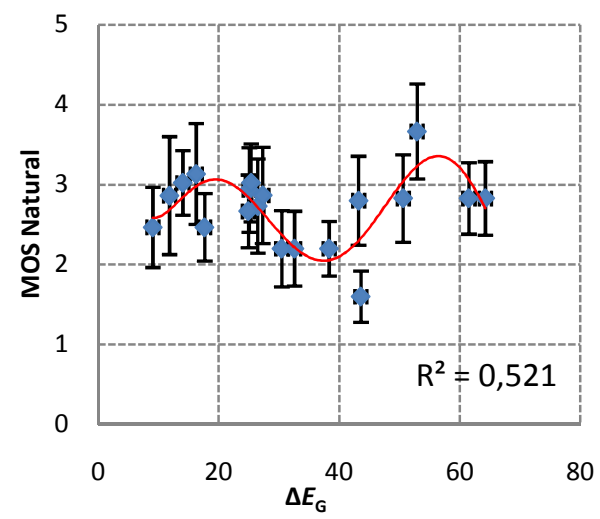

a. Corrélation entre $\triangle E_{G}$ et MOS Natural.

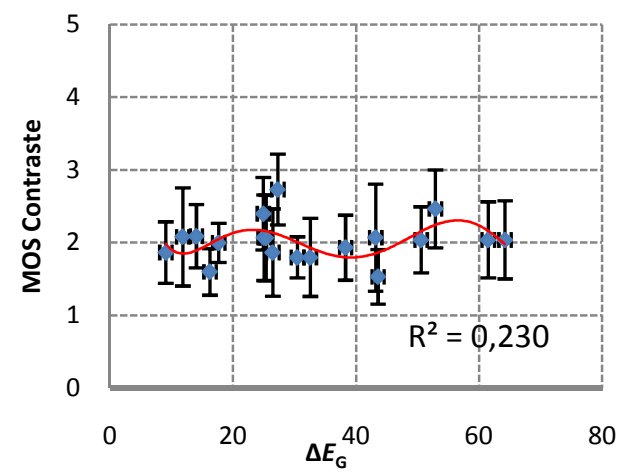

c. Corrélation entre $\Delta E_{G}$ et MOS Contraste.

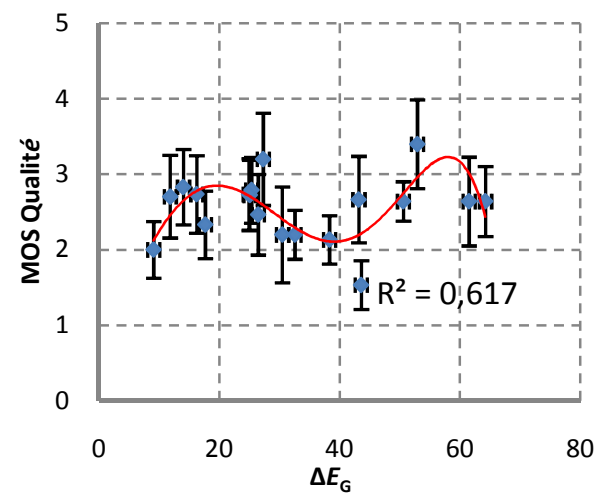

e. Corrélation entre $\Delta E_{G}$ et MOS Qualité.

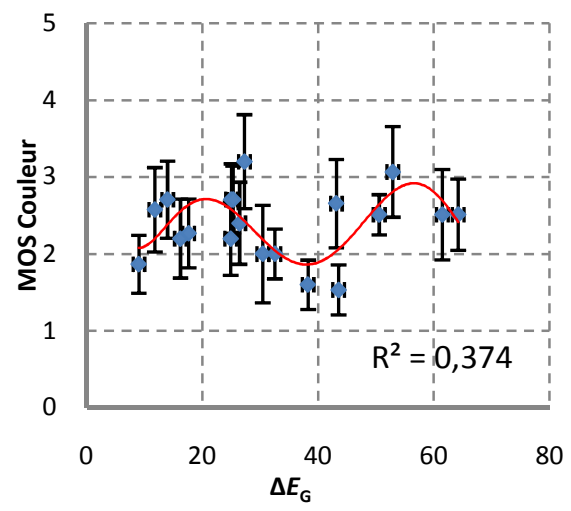

b. Corrélation entre $\Delta E_{G}$ et MOS Couleur.

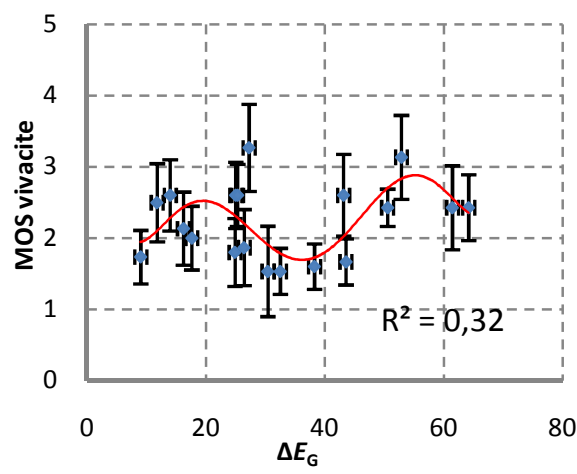

d. Corrélation entre $\triangle E_{G}$ et MOS Vivacité.

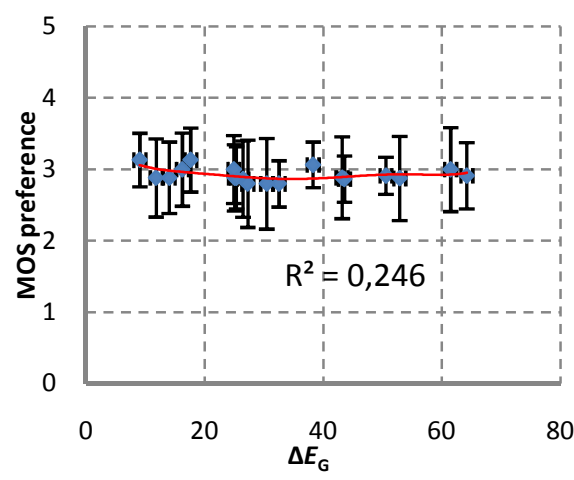

f. Corrélation entre $\Delta E_{G}$ et MOS préférence.

Figure 10. Corrélation entre Mean Opinion Scores (MOS) et le Delta $E_{G}$ Globale. 
Les résultats d'évaluation de la métrique sont illustrés sur figure 9 et figure 10. Pour chaque graphique, le coefficient de la corrélation totale $\mathrm{R}^{2}$ est calculé.

\begin{tabular}{|c|c|c|}
\hline $\begin{array}{c}\text { Coefficient de } \\
\text { corrélation } \mathbf{R}^{\mathbf{2}}\end{array}$ & $\Delta \mathbf{E}_{\mathbf{2 0 0 0}} / \mathbf{M O S}$ & $\Delta \mathbf{E}_{\mathbf{G}} / \mathbf{M O S}$ \\
\hline Contraste & 0,124 & 0,230 \\
\hline Natural & 0,204 & 0,521 \\
\hline Qualité & 0,255 & 0,617 \\
\hline Couleur & 0,271 & 0,374 \\
\hline Vivacité & 0,204 & 0,320 \\
\hline Préférence & 0,229 & 0,246 \\
\hline
\end{tabular}

Tab1. Corrélation entre MOS et les mesures objectives : $\Delta E_{2000}$ et $\Delta E_{G}$.

Une bonne mesure de la qualité objective devrait refléter la meilleure corrélation avec le MOS. Comme le prouve les résultats Tab1, le $\Delta \mathrm{E}_{2000}$ donne les plus mauvais valeurs de la corrélation pour les différentes caractéristiques comparées à la métrique proposée $\Delta \mathrm{E}_{\mathrm{G}}$. Nous obtenons de meilleurs résultats de la corrélation sur la diversité chromatique, le naturel et la qualité globale.

\section{Conclusion}

Dans ce papier, nous avons présenté une métrique de différence couleur basée sur une nouvelle approche pour simuler une partie des propriétés du SVH qui est la vision globale de l'image et non locale. L'originalité se trouve dans l'intégration de cette caractéristique et dans la réalisation d'une modélisation objective d'une sensation subjective. Cette étude a été basée sur des procédures d'évaluation objectives et subjectives prenant en compte la perception humaine. Les résultats obtenus sont très encourageants et ouvrent une voie prometteuse pour l'estimation de la qualité d'image couleur. Dans le contexte de la restauration d'images, cette métrique permettra de raffiner les algorithmes de correction automatique d'images en fonction des défauts présents dans les images.

\section{A R I M A}




\section{Bibliographie et biographie}

\subsection{Bibliographie}

[1] A.Tamtaoui, D. Aboutajdine. Mesure de la qualité visuelle des images compressées. Traitement du Signal 2003, 20(1).

[2] CIE. Methods to derive colour differences for images. Technical report. CIE, 2000.

[3] D. Nickerson. Munsell renotations used to study color spaces of hunter and adams. Journal ofoptic al society ofameric a, 40(2):85-88, 1950.

[4] D.-H. Kim and J. H. Nobbs. New weighting functions for weighted CIELAB colour difference formulae. 446-449, Kyoto, 1997.

[5] F.J.J. Clarke, R. McDonald, and D. Rigg. Modification to the JPC 79 colour difference formula. Journal of Society of Dyers Colour, 100(4) : 128-132, 1984.

[6] Hill, B., Roger, T., and Vorhagen, F. W.. Comparative analysis of the quantization of color spaces on the basis of the CIELAB color-difference formula. ACM Trans. Graph. 16(2): 109-154, 1997.

[7] K. McLaren. An introduction to instrumental shade passing and sorting and a review of recent developments. Journal of Society of Dyers Colour, 92(9):317-326, 1976.

[8] M. Chambah, A. Rizzi, C. Saint Jean, "Image quality and automatic color equalization", SPIE/IS\&T Electronic Imaging 2007, San Jose, California (USA).

[9] M.R. Luo and B. Rigg. BDF(1 :c) colour difference formula. Part 2 - performance of the formula. Journal of Society of Dyers Colour, 103(3) :126-132, 1987.

[10] M.R. Luo, G. Cui, and B. Rigg. Derivation of a rotation fuction for the new CIE colour difference formula. Proceedings of colour and visual scales 2000, 2000.

[11] P. C. COSMAN, R. M. GRAY and R. O. OLSHEN, Evaluating quality of compressed medical images: SNR, Subjective Rating, and Diagnostic Accuracy, Proceedings of the IEEE, Vol. 82(6), June 1994.

[12] P. Marziliano, F. Dufaux, S. Winkler, and T. Ebrahimi. Perceptual blur and ringing metrics: application to jpeg2000. Signal Processing: Image Communication, 19:163-172, 2004.

[13] R. McDonald. Acceptability decisions using the CMC color difference formula. Textiles chemist and colorist, 20(6) :31-36, 1988.

[14] R. McDonald. The effect of non-uniformity in the ANLAB color space on the interpretation of visual color differences. Journal of society of Dyers Colour, 90(6) :189198, 1974.

[15] R.S. Berns, D.H. Alman, L. Reniff, G.D. Snyder, and M. Balonon-Rosen. Visual determination of suprathreshold color difference tolerances using probit analysis. Color research and Application, 16 :297-316, 1991.

[16] Rec. ITU-R BT.500-11. Methodology for the subjective assessment of the quality of television pictures. ITU-R, 1974-2002.

[17] S. OLSSON, M. STROPPIANA, J. BAÏNA, Objective methods for assessment of video quality: state of the art, IEEE Transaction on Broadcasting, Vol. 43(4), December 1997.

[18] S. Ouni, M. Herbin, E. Zagrouba, Are existing procedures enough? Image and video quality assessment: review of subjective and objective metrics. SPIE/IS\&T Electronic Imaging, Proc. SPIE 6808, 68080Q, 28-30 January 2008, California, USA. 
[19] Z. Wang, A. C. Bovik and L. Lu, "Why is image quality assessment so difficult?" Proc. IEEE Int. Conf. Acoustics, Speech, and Signal Proc., vol. 4, pp. 3313-3316, May 2002.

\subsection{Biographie}

S. Ouni a obtenu son diplôme de master à la Faculté des Sciences de Tunis 2005. Elle est actuellement doctorante en cotutelle entre la Faculté des Sciences de Tunis et l'Université de Reims Champagne Ardenne. Elle est actuellement enseignante à la Faculté des Sciences de Tunis (FST). Ses activités de recherche s'articulent essentiellement autour de l'évaluation de la qualité des images couleurs.

E.Zagrouba est actuellement professeur à l'Institut Supérieur d'Informatique (ISI) de Tunis. Il a obtenu son doctorat à l'institut National Polytechnique de Toulouse (INPT) en 1994 puis son habilitation universitaire à la Faculté des Sciences de Tunis en 2005. Il est responsable de l'équipe de recherche SIVREC dont les activités majeures sont axées sur le traitement d'images et la vision par ordinateur.

M.Chambah est actuellement maitre de conférences à l'IUT de Reims Charleville. Il a obtenu son doctorat à l'Université de La Rochelle (France). Il est membre de l'équipe de recherche Crestic dont les activités majeures sont axées sur la restauration numérique des films couleur.

M.Herbin est actuellement professeur à l'IUT de Reims Châlons Charleville. Il est membre de l'équipe de recherche Crestic à l'Université de Reims Champagne Ardenne dont les activités majeures sont axées sur le traitement d'images. 